\title{
Using Target Engagement Biomarkers to Predict Clinical Efficacy of MetAP2 Inhibitors
}

\author{
Pamela J. Farrell, Christopher J. Zopf, ${ }^{1}$ (DHuey-Jing Huang, Deepika Balakrishna, \\ Corine Holub, James Bilakovics, Andrea Fanjul, Jennifer Matuszkiewicz, Artur Plonowski, ${ }^{2}$ \\ Paul Rolzin, Urmi Banerjee, Jacques Ermolieff, Zacharia S. Cheruvallath, \\ Christopher McBride, Darian Bartkowski, Crystal Mazur, Alok Pachori, \\ and Christopher J. Larson ${ }^{3}$
}

Biological Sciences (P.J.F., H.-J.H., De.B., C.H., J.B., A.F., J.M., A.PI., P.R., U.B., J.E., C.J.L.), Chemistry (Z.S.C., C.Mc.), and Drug Metabolism and Pharmacokinetics (Da.B.), Takeda California, San Diego, California; Modeling and Simulation, Takeda Boston, Cambridge, Massachusetts (C.J.Z.); and Translational Research Institute for Metabolism and Diabetes, Florida Hospital Campus, Orlando, Florida (C.Ma., A.Pa.)

Received May 11, 2019; accepted September 4, 2019

\section{ABSTRACT}

Target-engagement pharmacodynamic (PD) biomarkers are valuable tools in the prioritization of drug candidates, especially for novel, first-in-class mechanisms whose robustness to alter disease outcome is unknown. Methionine aminopeptidase 2 (MetAP2) is a cytosolic metalloenzyme that cleaves the $\mathrm{N}$-terminal methionine from nascent proteins. Inhibition of MetAP2 leads to weight loss in obese rodents, dogs and humans. However, there is a need to develop efficacious compounds that specifically inhibit MetAP2 with an improved safety profile. The objective of this study was to identify a PD biomarker for selecting potent, efficacious compounds and for predicting clinical efficacy that would result from inhibition of MetAP2. Here we report the use of NMet14-3-3 $\gamma$ for this purpose. Treatment of primary human cells with MetAP2 inhibitors resulted in an approx. 10-fold increase in NMet14-3$3 \gamma$ levels. Furthermore, treatment of diet-induced obese mice with these compounds reduced body weight (approx. 20\%) and increased NMet14-3-3 $\gamma$ (approx. 15-fold) in adipose tissues. The effects on target engagement and body weight increased over time and were dependent on dose and administration frequency of compound. The relationship between compound concentration in plasma, NMet14-3-3 $\gamma$ in tissue, and reduction of body weight in obese mice was used to generate a pharmacokineticpharmacodynamic-efficacy model for predicting efficacy of
MetAP2 inhibitors in mice. We also developed a model for predicting weight loss in humans using a target engagement PD assay that measures inhibitor-bound MetAP2 in blood. In summary, MetAP2 target engagement biomarkers can be used to select efficacious compounds and predict weight loss in humans.

\section{SIGNIFICANCE STATEMENT}

The application of target engagement pharmacodynamic biomarkers during drug development provides a means to determine the dose required to fully engage the intended target and an approach to connect the drug target to physiological effects. This work exemplifies the process of using target engagement biomarkers during preclinical research to select new drug candidates and predict clinical efficacy. We determine concentration of MetAP2 antiobesity compounds needed to produce pharmacological activity in primary human cells and in target tissues from an appropriate animal model and establish key relationships between pharmacokinetics, pharmacodynamics, and efficacy, including the duration of effects after drug administration. The biomarkers described here can aid decision-making in early clinical trials of MetAP2 inhibitors for the treatment of obesity.
This work was supported and funded by Takeda Pharmaceuticals

${ }^{1}$ Cowen and Co., Boston, Massachusetts.

${ }^{2}$ Current affiliation: Seal Rock Therapeutics, Cupertino, California.

${ }^{3}$ Sanford Burnham Prebys Medical Discovery Institute, San Diego, California. https://doi.org/10.1124/jpet.119.259028.

S This article has supplemental material available at jpet.aspetjournals.org.

\section{Introduction}

Obesity is a major risk factor for diabetes, heart disease, stroke, hepatic steatosis, cancer, and numerous other medical conditions. Although the health issues associated with obesity are well established, the obesity rate continues to rise, with prevalence at $36 \%$ of US adults and $17 \%$ of US children (Ogden et al., 2015). Pharmacological treatment options for obesity are limited and exhibit modest efficacy. The top

ABBREVIATIONS: AUC, area under the curve; BAT, brown adipose tissue; Brij-35 is a nonionic polyoxyethylene surfactant BWL, body weight loss; $\mathrm{DIO}$, diet-induced obese; DMSO, dimethyl sulfoxide; $\mathrm{E}$, efficacy; $\mathrm{EC}_{50}$, half-maximal effective concentration; $\mathrm{IC}_{50}$, half-maximal inhibitory concentration; Met-AMC Methionine-7-amido-4-methylcoumarin MetAP2, methionine aminopeptidase; NMet14-3-3 $\gamma$, 14-3-3 $\gamma$ protein containing methionine at the amino terminus; PBMC, peripheral blood mononuclear cells; PD, pharmacodynamics; PK, pharmacokinetics; QD, daily; TCEP, $3,3^{\prime}, 3^{\prime \prime}$-phosphanetriyltripropanoic acid. 
drugs produce 5\% weight loss over 3 months (Yanovski and Yanovski, 2014). This moderate effect as well as lack of sustained long-term response falls far short of patient expectations (Foster et al., 1997). Although bariatric surgery is a more effective option for weight loss, many patients do not qualify or do not prefer surgical intervention. Hence, there is a need for more robust and safer antiobesity medications.

Development of novel drugs requires use of a biomarker strategy to make important go/no go decisions. Pharmacodynamic biomarkers are often used to assess the efficacy of novel compounds but determining whether a drug interacts with its specific target is very difficult using distal biomarkers, as converging signals can influence biomarker response. Target engagement biomarkers that are proximal to the drug target can be used to determine the dose required to fully engage the intended target and connect the drug target to physiologic effects. For example, a target engagement biomarker that directly measures drug occupancy on the drug target can be used to understand if partial target occupancy could cause sufficient response in patients. Target engagement biomarkers can also be used to understand the level of occupancy that causes saturation of a desired downstream effect. This information can then be used to determine the appropriate dose schedule and is especially helpful in making go/no go decisions for drugs that have a novel or unknown mechanism.

The methionine aminopeptidase (MetAP2) inhibitor class of drugs has shown antiobesity efficacy in clinical trials, with up to $10.6 \%$ weight loss over 3 months reported for beloranib (Hughes et al., 2013; Kim et al., 2015). MetAP2 expression is increased 1.2- to 1.95-fold $(P<0.03)$ in adipocytes from obese humans and obese mice (Baranova et al., 2005; Hageman et al., 2010; Klimcakova et al., 2011; Rosell et al., 2014). Although the details of the molecular mechanism are not well defined, inhibitors of MetAP2 act on fat metabolism pathways, stimulating lipolysis and reducing fatty acid synthesis (Rupnick et al., 2002; Lijnen et al., 2010). Several studies have demonstrated positive association between weight loss by MetAP2 inhibition and reduction in adipose mass in preclinical models of obesity (Rupnick et al., 2002; Brakenhielm et al., 2004; Kim et al., 2007; Lijnen et al., 2010; White et al., 2012). Furthermore, loss of adipose mass has been demonstrated in clinical trials with first generation MetAP2 inhibitors (Hughes et al., 2013; Kim et al., 2015), supporting the adipose reduction mechanism. However, subcutaneous route of administration and dose-limiting adverse effects have hindered the development and progression of these first generation compounds (https://zafgen.gcs-web.com/news-releases/ news-release-details/zafgen-refocuses-resources-developmentdifferentiated-second).

Because MetAP2 inhibitors have shown potential for adipose weight reduction in obese individuals, we pursued studies to develop potent chemical classes of these compounds with improved drug profile. To select candidate drugs we developed a translational strategy for predicting body weight loss (BWL) caused by MetAP2 inhibitors. We performed in vitro and in vivo experiments to evaluate the extent and duration of MetAP2 target engagement that correlates with BWL efficacy. Our studies reveal that changes in NMet14-3$3 \gamma$, a MetAP2 substrate, or change in level of MetAP2 occupied by inhibitor can be used to predict the final BWL efficacy of MetAP2 drug candidates. The NMet14-3-3 $\gamma$ PD biomarker and MetAP2 occupancy results are used to establish pharmacokinetic-pharmacodynamic-efficacy (PK-PD-E) relationships for MetAP2 inhibitors and develop translational models for predicting efficacy in clinical studies. This work exemplifies the process of using target engagement biomarkers during preclinical research to select new drug candidates, predict efficacy, and ultimately determine a dosage regimen for clinical trials.

\section{Materials and Methods}

Reagents. Small-molecule compounds were synthesized at Takeda California, San Diego, CA, as described in Cheruvallath et al., (2016) and McBride et al., (2016). For in vitro studies, compounds were solvated in dimethyl sulfoxide (DMSO) and for in vivo studies compound 1 was formulated in $0.5 \%$ methylcellulose; beloranib was formulated in $2 \%$ captisol $/ 0.3 \%$ citric acid, and A357300 was formulated in $0.2 \%$ hydroxypropylmethyl cellulose. Met-AMC, a fluorescent MetAP2 substrate, was purchased at Enzo Life Sciences. $\mathrm{CoCl}_{2}$ and $\mathrm{MnCl}_{2}$ solutions were purchased at Sigma-Aldrich. The following antibodies were purchased from the indicated vendors: NMet14-3-3 $\gamma$ (Novus), total 14-3-3 $\gamma$ antibody (Cell Signaling), beta-actin (Sigma), glyceraldehyde3-phosphate dehydrogenase (GAPDH; US Biologic), and MetAP2 (Santa Cruz Biotechnology).

Enzyme Activity. Each recombinant human MetAP enzyme was expressed in Sf9 cells and purified in the presence of $\mathrm{Mn}^{+2}$ or $\mathrm{Co}^{+2}$. For the determination of half-maximal inhibitory concentration $\left(\mathrm{IC}_{50}\right)$, the MetAP2 enzyme activity was measured using a fluorogenic substrate, Met-AMC, in an assay buffer containing $50 \mathrm{mM}$ Hepes, $100 \mathrm{mM} \mathrm{NaCl}$, $0.005 \%$ Brij35, $1 \mathrm{mM}$ TCEP, $0.1 \mathrm{mg} / \mathrm{ml}$ bovine serum albumin, and $10 \mu \mathrm{M} \mathrm{MnCl}_{2}$ or $10 \mu \mathrm{M} \mathrm{CoCl}_{2}$ and $1 \% \mathrm{DMSO}$ at $\mathrm{pH}$ 7.5. Each assay was conducted by adding a serial dilution of inhibitor to a solution containing $20 \mathrm{nM}$ enzyme and 100 or $30 \mu \mathrm{M}$ Met-AMC substrate in the presence of $\mathrm{Mn}^{+2}$ or $\mathrm{Co}^{+2}$, respectively. MetAP1 assays were conducted likewise, except $100 \mathrm{nM}$ enzyme and $100 \mu \mathrm{M}$ of Met-AMC substrate were used. For determination of $k_{\text {inact }} / K_{i}$, a mixture containing inhibitor (serial dilution) and substrate $(200 \mu \mathrm{M})$ was prepared in the assay buffer. To initiate the reaction MetAP2 was added to a final $20 \mathrm{nM}$ concentration. Reaction progress curves showing timedependent onset of inhibition were monitored by recording the product appearance with time, and experimental data were curve-fitted to determine the pseudo first-order rate constant, $\mathrm{k}_{\mathrm{obs}}$, for each inhibitor concentration (Morrison, 1982).

The selectivity of compounds was evaluated against a panel 26 proteases at compound concentration range of $5 \mathrm{nM}$ to $10 \mu \mathrm{M}$ by Reaction Biology Corporation (Malvern, PA). Proteases tested included: ADAM9, ADAM17, caspase1, cathepsin C, cathepsin D, cathepsin G, cathepsin S, DPP4, factor10a, granzyme B, MMP1, MMP2, MMP3, MMP7, MMP8, MMP9, MMP10, MMP12, MMP13, MMP14, papain, proteinase K, TACE, thrombin, tryptase $\gamma 1$, and trypsin. The assay conditions were according to vendor specifications.

MetAP2 Target Engagement in Primary Human Cells. HUVEC cells (Lonza) were seeded in 96-well tissue culture microplates and cultured for 24 hours prior to addition of compounds. Cells were incubated with compound for 24 hours, then media was removed and cell extracts were prepared by adding $50 \mu \mathrm{l}$ of cell lysis buffer (Cell Signaling). Insoluble material was removed by centrifugation and samples were boiled for 5 minutes in gel loading buffer. Positive and negative control samples were included in each study for quality control. These included cells treated with DMSO vehicle for the negative control, cells treated with saturating concentrations of MetAP2 inhibitor (e.g., $10 \mu \mathrm{M}$ A357300 or $100 \mathrm{nM}$ beloranib) for the positive control, and HeLa cells, which have significant levels of endogenous NMet14-3-3 $\gamma$. Proteins were resolved by SDS-PAGE and transferred to polyvinylidene fluoride membrane. Membranes were blocked with Odyssey buffer (LI-COR Biotechnology) then incubated with the NMet14-3-3 $\gamma$ antibody and control antibodies (beta-actin or 
GAPDH or 14-3-3 $\gamma$, as indicated in figures), followed by incubation with the appropriate secondary antibodies, IR-Dye $680 \mathrm{CW}$ - or $800 \mathrm{CW}$ conjugated antibodies (LI-COR Biotechnology). To increase sensitivity in the HUVEC assay, we diluted primary antibodies in Can Get Solution 1 (Toyobo). Membranes were scanned, and integrated band intensities corresponding to each protein were quantified using LICOR Biotechnology software. Compound $\mathrm{IC}_{50}$ s were obtained by curve-fitting the ratio of NMet14-3-3 $\gamma$ protein signal to control protein (see above and $y$-axis of graphs in figures) divided by the ratio determined for the DMSO vehicle control. A similar Western blotting procedure was used for human peripheral blood mononuclear cells (PBMCs), which were isolated from donors immediately after blood was drawn.

Mouse Models and In Vivo Procedures. All animal studies were in accordance with Institutional Animal Care and Use Committee. C57BL/6 male mice were obtained from Jackson Laboratories and fed a $60 \%$ fat diet (HFD, D12492; Research Diets) to obtain dietinduced obese (DIO) mice (see Supplemental Methods). At the end of study tissues were dissected, immediately frozen, and stored at $-80^{\circ} \mathrm{C}$ in tissue lysis tubes with matrix A (MP Biomedicals). For detection of NMet14-3-3 $\gamma$ in tissues, the fast prep bead beater (Biospec Products, Inc.) was used to homogenize tissue in $2 \%$ SDS lysis buffer, and $15 \mu \mathrm{g}$ of protein was loaded per lane for gel electrophoresis followed by immunoblotting as outlined above.

For PK analysis, plasma was collected via centrifugation of blood, then stored at $-80^{\circ} \mathrm{C}$. Plasma compound level was analyzed using a research-grade liquid chromatography-tandem mass spectrometry assay. $\mathrm{PK}$ parameters [maximum concentration $\left(\mathrm{C}_{\max }\right)$, terminal halflife $\left(t_{1 / 2}\right)$, systemic clearance (CL), area under the curve extrapolated to infinity $\left(\mathrm{AUC}_{\text {extrap }}\right)$ or to latest time drug is detected $\left.\left(\mathrm{AUC}_{0-\mathrm{t}}\right)\right]$ were determined with the Phoenix WinNonlin software (Pharsight, CA). Unbound (free) drug was estimated using the species-specific percent protein binding, determined by equilibrium dialysis.

For the occupancy assay, which detects "free" unbound MetAP2 (as opposed to "occupied" inhibitor-bound MetAP2), we quantified free MetAP2 in blood from mice treated with beloranib. MetAP2 in the blood from these mice can exist as either beloranib-bound MetAP2 or free MetAP2. Because beloranib covalently binds to MetAP2, the beloranib-bound MetAP2 remains stable throughout blood collection and lysis, and the free MetAP2 can be captured by adding a fumagillin analog probe to the lysate. This previously described probe (Vath, 2010; Zhou et al., 2013) was designed to covalently bind to MetAP2 at the same site as beloranib and also contains a biotin tag. As such, the probe-bound MetAP2, which represents free MetAP2, can be isolated on streptavidin plates and subsequently quantified as follows. To perform the occupancy analysis, $200 \mu \mathrm{l}$ of blood from treated mice was dispensed into lithium heparinized collection tubes. Red blood cells were lysed by adding $800 \mu \mathrm{l}$ ammonium chloride solution. Samples were then vortexed, centrifuged, washed, and cell pellets kept frozen at $-80^{\circ} \mathrm{C}$. Each pellet was dissolved in $100 \mu \mathrm{l}$ of lysis buffer (MesoScale Discovery, MSD) containing $30 \mathrm{nM}$ concentration of a biotinylated fumagillin analog probe (Vath, 2010; Zhou et al., 2013) and incubated on an orbital shaker for 1 hours. Samples were centrifuged to remove insoluble material, and $50 \mu \mathrm{l}$ of supernatant was transferred to a blocked streptavidin gold ELISA plate (MSD). Subsequently, $50 \mu \mathrm{l}$ of $4 \mu \mathrm{g} / \mathrm{ml}$ MetAP2 antibody solution was added to all wells followed by $50 \mu \mathrm{l}$ of $10 \mu \mathrm{g} / \mathrm{ml}$ sulfo-tag secondary antibody (MSD). Plates were washed, then scanned immediately after the addition of read buffer (MSD). The following control samples were included in triplicate with each assay: naive blood treated ex vivo with vehicle or $30 \mathrm{nM}$ beloranib. Control samples were incubated for 30 minutes with shaking, and the occupancy assay performed as described above. These controls were used to calculate percentage of free unbound MetAP2 versus inhibitor-bound MetAP2 in blood from treated mice. To determine the beloranib concentration to use for the $100 \%$ inhibitor-bound MetAP2, we performed the occupancy assay using samples of various concentrations of beloranib added ex vivo to naive blood.
PK-PD-E Modeling. The details of the PK-PD-E modeling of compound 1 and beloranib can be found in the Supplemental Methods. Supplementary tables and figures showing the models for compound 1 include Supplemental Table 1 and Supplemental Figs. 1 and 2 and for beloranib include Supplemental Table 1 and Supplemental Figs. 3 and 4. Briefly, the PK model for each compound in DIO mice was built using the plasma concentrations at several doses. The models were fit simultaneously to all dose groups (Supplemental Table 1 and Supplemental Figs. 1 and 3) using the appropriate built-in function in Phoenix WinNonlin (Certara USA, Princeton, NJ). The PK-PD model for compound 1 and NMet14-3-3 $\gamma$ response in DIO mice was built using the compound 1 PK model and NMet14-3-3 $\gamma$ levels in PBMCs following a single dose. Likewise, the PK-PD model for beloranib was built using the beloranib PK model and fraction of unbound MetAP2 in blood following a single dose. For each PK-PD model the response data were fit simultaneously across all dose groups using a naive-pooled approach and additive error model in Phoenix WinNonlin (Supplemental Figs. 2 and 4; Supplemental Table 1). The PK-PD model for compound 1 was then used to simulate NMet14-3-3 $\gamma$ levels through 28 days to predict peak biomarker accumulation following daily compound 1 treatment. The PK-E model for beloranib in DIO mice was built using the total plasma concentrations and the fraction of body weight change in mice after daily or biweekly dosing for 28 days. The response data were fit simultaneously across all dose groups using a naive-pooled approach in Phoenix WinNonlin (Supplemental Fig. 3).

Translation of Human Clinical Beloranib Exposures to Mouse Equivalent. Using the available clinical exposures to beloranib from phase 1 dose escalations in cancer patients and obese patients (Shin et al., 2010; Hughes et al., 2013), we constructed a doseexposure $\left(\mathrm{AUC}_{0-24 \mathrm{~h}}\right)$ relationship to estimate the human plasma concentration AUC achieved for a given dose (Supplemental Fig. 5). To translate a total human exposure to the mouse equivalent, we then corrected for species differences in plasma protein binding (81\% in human compared with $91 \%$ in mouse) to estimate the total exposure in mouse.

Statistics. Evaluation of the effects of compounds on body weight and target engagement in vivo is expressed as mean \pm S.E.M. Statistical significance was determined using two-way analysis of variance with Tukey's multiple comparisons test using GraphPad Prism software.

\section{Results}

Structurally Diverse Set of MetAP2 Inhibitors. We used a diverse set of MetAP2 inhibitors to evaluate the specific effects of MetAP2 inhibition, irrespective of chemical class. We selected two compounds that had been previously characterized, beloranib and A357300, as well as a compound from our distinct pyrazolo-indole chemical series (McBride et al., 2016). Figure 1 shows the chemical structure of each of these inhibitors. Beloranib, a fumagillin analog, forms a covalent bond with MetAP2, whereas compound 1 and A357300 bind reversibly to MetAP2. The mode of binding for each of these chemical classes of inhibitors to MetAP2 has been previously characterized using x-ray diffraction crystallography (Liu et al., 1998; Wang et al., 2003; McBride et al., 2016). Using purified recombinant enzymes (see Materials and Methods) we confirmed that all three compounds potently inhibit the enzymatic activity of MetAP2 with $\mathrm{IC}_{50}$ values within 3-fold of each other, ranging from 8.5 to $26 \mathrm{nM}$ (Table 1). In addition, very little to no activity was observed against MetAP1 and 26 other proteases for the three compounds $\left(\mathrm{IC}_{50}>10,000 \mathrm{nM}\right)$.

Target Engagement Activity of MetAP2 Inhibitors in Primary Human Cells. We assessed MetAP2 target engagement 


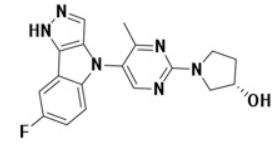

Compound 1

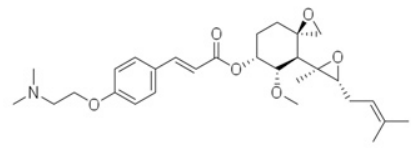

Beloranib

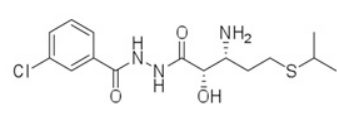

A357300
Fig. 1. Structures of three chemically diverse MetAP2 inhibitors used in our studies. Compound 1 was discovered by Takeda and is described in McBride et al. (2016). Beloranib was discovered by Chong Kun Dang and later developed by Zafgen (Morgen et al., 2016). Beloranib is also known as CKD-732 and ZGN-433 and is described in Hong et al. (2000). A357300 was discovered by Abbott and is described in Wang et al. (2003). by inhibitor in cells by measuring levels of a MetAP2 substrate, NMet14-3-3 $\gamma$. HUVECs or freshly isolated human PBMCs were seeded in 96-well plates and treated with various concentrations of compound 1, A357300, or beloranib. As shown in Fig. 2, each compound caused a dose-dependent induction of cellular NMet14-3$3 \gamma$. The half-maximal effective concentration $\left(\mathrm{EC}_{50}\right)$ values in HUVECs and human PBMCs were calculated for the compounds using dose-response curves obtained from each cell type (Fig. 2). Beloranib, which binds covalently to MetAP2 exhibited very high potency in cells ( $<1 \mathrm{nM}$ ), whereas A357300 and compound 1, which bind reversibly to MetAP2, exhibited average cellular potency of approx. 10 and $30 \mathrm{nM}$, respectively. We performed two additional in vitro studies with these compounds to aide in our translational analyses. First, for mouse-to-human translation we determined that MetAP2 inhibitors had similar activity in cells from each species (Supplemental Table 2). Second, for lean to obese translation we evaluated compound potency in cells from both lean and obese subjects and found similar activity for individuals with BMI ranging from 19 to 44 (Supplemental Table 3). Overall, our in vitro analysis confirmed that these three chemically diverse MetAP2 compounds each potently inhibit purified MetAP2 enzyme and inhibit MetAP2 in cells derived from a variety of sources.

In Vivo Target Engagement Pharmacodynamics, Pharmacokinetics, and Efficacy. Next, we determined MetAP2 target engagement in a diet-induced obesity mouse model using compound 1 , a reversible inhibitor from the pyrazolo-indole chemical series. This compound was selected for further analysis because of its overall favorable in vitro properties, including potent cellular target engagement, selectivity profile across proteases, compound stability in microsome, and cytochrome P450 assays, as well as performance in a panel of in vitro toxicology assays (McBride et al., 2016). To determine the appropriate dose range of compound 1 in vivo, we measured plasma levels of the compound after oral administration of a range of doses to cohorts of DIO mice (Fig. 3A). At 2 hours post-drug administration for all three doses, compound 1 was at levels expected to inhibit, at least intermittently, MetAP2 in vivo on the basis of its cellular potency and nonspecific protein binding (91\%). Because adipose is a key target tissue that may mediate the efficacy of MetAP2 inhibitors (Rupnick et al., 2002; Lijnen et al., 2010;
Vath, 2010; White et al., 2012; Huang et al., 2019), we measured levels of NMet14-3-3 $\gamma$ in adipose tissue from obese mice treated with compound 1. As illustrated in Fig. 3B, a dose-dependent increase in NMet14-3-3 $\gamma$ levels in subcutaneous white adipose tissue (scWAT) and brown adipose tissue (BAT) was observed in DIO mice treated once daily for 2 weeks with compound 1 . The weight loss corresponding to each dose was measured prior to adipose collection and it was plotted versus NMet14-3-3 $\gamma$ levels in adipose at various doses of compound 1 (Fig. 3C). These initial PK-PD-E studies provided insight into the dose selection for a more extensive analysis of target engagement over time.

We investigated the PK-PD relationship over time by measuring drug levels and target engagement in PBMCs of obese mice treated with a single dose of compound 1 (Fig. 4, A and B). Compound concentration was plotted over time and AUC values were determined for each dose. As shown in Fig. 4, the 10-, 30-, and 100-mg/kg doses resulted in plasma AUC levels of 24,103 , and $353 \mu \mathrm{g} *$ hour/ml, respectively (Fig. 4A). For the corresponding PD analysis, we first measured NMet14-3-3 $\gamma$ in PBMCs and BAT over time (Supplemental Fig. 6). We selected BAT since MetAP2 inhibitors produce direct effects on brown adipocytes in vitro, consistent with their robust effects on target engagement and fatty acid metabolites in this adipose depot in vivo (Huang et al., 2019). Similar target engagement was observed in PBMCs and BAT over time, suggesting NMet14-3-3 $\gamma$ in PBMCs could serve as a less invasive surrogate peripheral biomarker. Subsequently, we determined target engagement over time in PBMCs isolated from mice treated with various doses. As shown in Fig. 4B levels of NMet14-3-3 $\gamma$ reach peak levels at 36-48 hours and return to baseline at 72-84 hours after each dose. It is noteworthy that the NMet14-3-3 $\gamma$ increase takes time to develop (compare time courses in Fig. 4, A and B).

Next, to investigate how the PK and PD are related to body weight loss, we conducted efficacy studies over 2-4 weeks at several doses. Daily oral administration resulted in dosedependent weight reduction, which became apparent after 48 hours of compound administration and continued to progress as compound was dosed daily for 4 weeks (Fig. 4C).

\section{TABLE 1}

Enzyme inhibitory activity of MetAP2 compounds

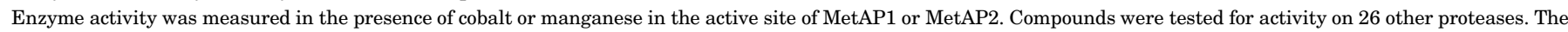
highest concentration used in each analysis was 10 or $100 \mu \mathrm{M}$.

\begin{tabular}{|c|c|c|c|c|}
\hline & $\operatorname{MetAP2}(\mathrm{Co} ; \mathrm{Mn}) \mathrm{IC}_{50}$ & $\operatorname{MetAP2}(\mathrm{Mn}) \mathrm{K}_{\text {inact }}(1 / \mathrm{min}) \div \mathrm{K}_{\mathrm{i}}$ & MetAP1 $(\mathrm{Co} ; \mathrm{Mn}) \mathrm{IC}_{50}$ & 26 Proteases $\mathrm{IC}_{50}$ \\
\hline & $n M$ & $\mu M$ & $n M$ & $n M$ \\
\hline Compound 1 & $10 \pm 6 ; 8.5 \pm 4$ & ND & $>100,000 ;>100,000$ & $>10,000$ \\
\hline Beloranib & ${ }^{a} 8.2 \pm 0.4 ;{ }^{a} 26 \pm 8$ & ${ }^{a} 0.7 \pm 0.2$ & $>10,000 ;>10,000$ & $>10,000$ \\
\hline A357300 & $20 \pm 11 ;$ nd & ND & 18,$000 ; 33,000$ & $>10,000$ \\
\hline
\end{tabular}

ND, not determined.

${ }^{a}$ Because beloranib binds covalently to MetAP2, the more relevant value to describe potency is the rate of inactivation $\left(\mathrm{K}_{\text {inact }} / \mathrm{K}_{\mathrm{i}}\right)$. 
A

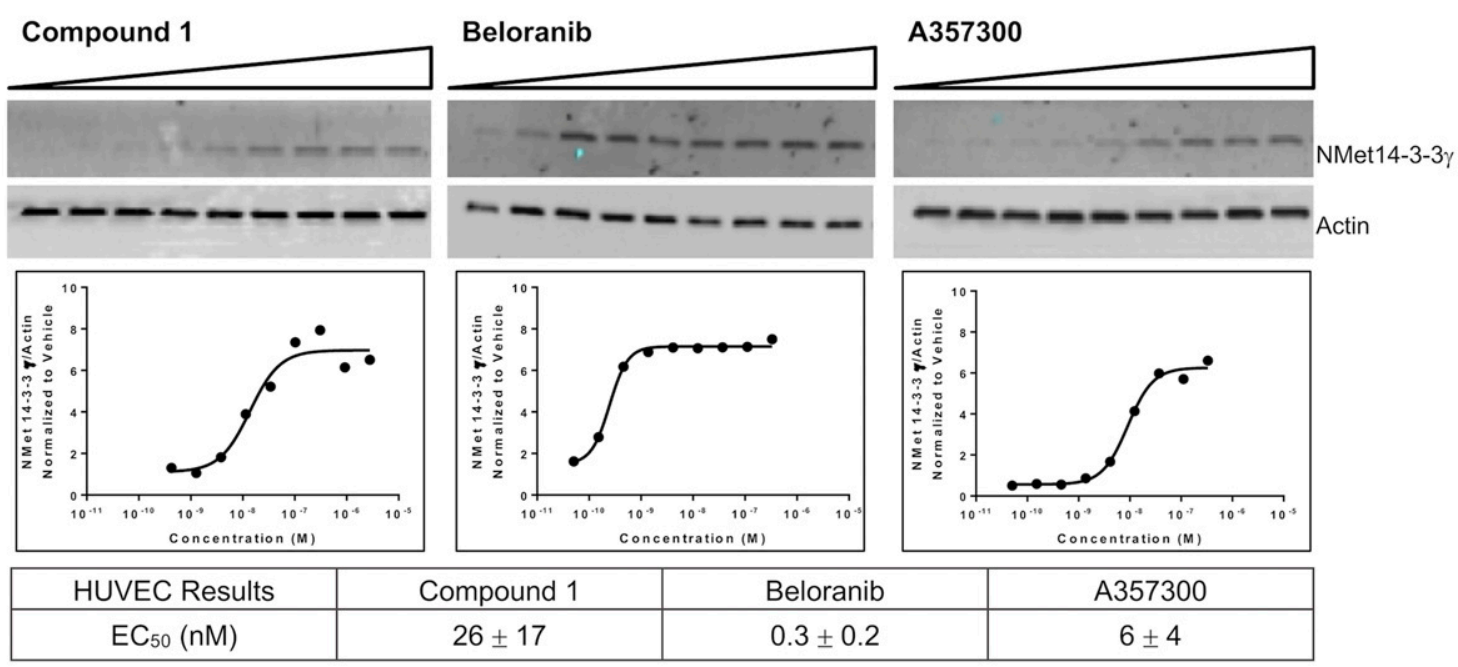

B $\quad$ ड

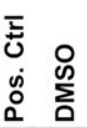

Fig. 2. Target engagement activity of MetAP2 inhibitors in primary human endothelial cells and primary human PBMCs. Representative NMet14-3-3 $\gamma$ Western blots (top), dose-response curves (middle), and $\mathrm{EC}_{50} \mathrm{~s}$ are shown for three MetAP2 inhibitors in primary human endothelial cells (A) and freshly isolated human PBMCs (B). For PBMCs each donor is represented by a different color curve. The target engagement assay was developed as a medium throughput assay for compound screening, and $\mathrm{EC}_{50}$ s shown represent the average of all values $(n \geq 3)$ obtained during the drug discovery campaign.

A plateau was reached between 2 and 4 weeks with lower doses reaching steady-state weight loss earlier. At 2 weeks the body weight reduction was $1 \% \pm 1 \%, 5 \% \pm 1 \%, 11 \% \pm 3 \%$, and $22 \% \pm 2 \%$ for the $3-, 10-, 30-$, and $100-\mathrm{mg} / \mathrm{kg}$ doses, respectively (Fig. 4C, day 14). To compare the longer-term efficacy studies to target engagement PD, a PK-PD model was generated. We used the compound 1 concentration data shown in Fig. 4A and the NMet14-3-3 $\gamma$ data shown in Fig. 4B to generate a PK model and a dynamic PK-PD model (Materials and Methods, Supplemental Figs. 1 and 2; Supplemental Table 1). The PK-PD model was then used to simulate the steady-state NMet14-3-3 $\gamma$ over 4 weeks of daily dosing for each dose level (Fig. 4D). The model shows PD reaching a plateau over time, similar to body weight efficacy but earlier in time.
To further evaluate the PD-E relationship, we determined if the data from a single-dose PD study could be used to predict longer-term efficacy, an approach that could be applied in the clinic. Initially, to investigate this we calculated the area under the effect curve (AUE) at several dose levels of compound 1 (e.g., area under PD time-course curves shown in Fig. 4B) and plotted it versus the corresponding dose. The dose-response curve (fold increase of NMet14-3-3 $\gamma$ AUE vs. vehicle) is shown in Fig. 5A. On the same graph we plotted BWL efficacy versus dose (Fig. 5A, square symbols). The doseresponse curves show relationships between efficacy at day 14 and NMet14-3-3 $\gamma$ levels detected on day 1 after one dose, thus suggesting that PD measured after a single dose could be used to predict steady-state efficacy after daily dosing. For example, an efficacy of 5\% BWL correlates with an approx. 5-fold 
A

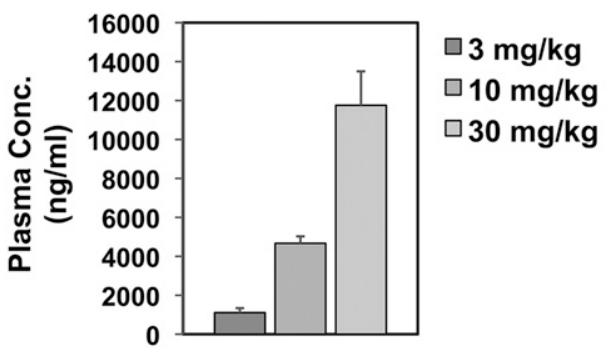

B

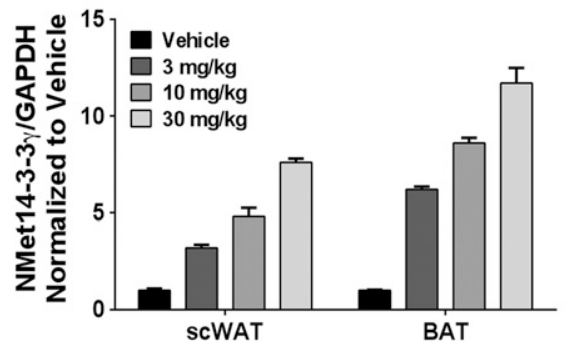

C

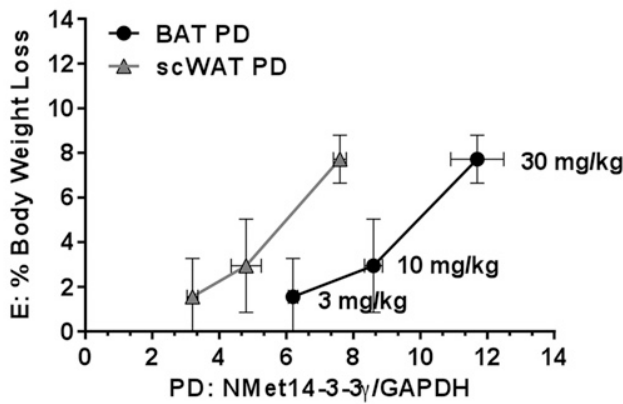

Fig. 3. Target engagement activity of compound 1 in vivo. (A) Plasma levels of compound 1-2 hours after oral administration of compound at the indicated dose ( $n=3$ mice per group). (B) Dose-dependent effect of compound 1 on levels of NMet14-3-3 $\gamma$ in adipose tissues from DIO mice after 2 weeks of daily oral treatment ( $n=5$ mice per group). Subcutaneous white adipose tissue (scWAT) and BAT were collected 2 hours after the 15th dose and evaluated for MetAP2 target engagement (see Materials and Methods). (C) PD-E plot showing the correlation of MetAP2 target engagement in adipose and BWL determined at the end of the study (day 15). PD-E curve for BAT contains circle symbols and the PD-E curve for WAT contains triangle symbols.

increase in target engagement, and an efficacy of $10 \%$ BWL correlates with an approx. 10-fold increase in target engagement. This analysis also allows an evaluation of minimum target engagement needed for BWL. For example, at $1-3 \mathrm{mg} / \mathrm{kg}$ of compound 1, the AUE for target engagement increased approx. 2.5-fold; however, this dose was not sufficient to cause BWL, suggesting a threshold for target engagement may be required for efficacy. Finally, to compare PD and $\mathrm{E}$ across the full treatment duration, BWL on day 14 was plotted versus the NMet14-3-3 $\gamma$ simulated over the same time period. This analysis showed a strong correlation between simulated target engagement and efficacy in mice (Fig. 5B) and provided additional evidence that MetAP2 target engagement measurements in PBMCs can be used to predict efficacy in mice.

To extend our findings to other chemical classes of MetAP2 inhibitors, PD-E studies were performed with compound 1, A357300, and beloranib. Using three chemicals with substantially different structures (Fig. 1), binding modes, and potencies increased the confidence that MetAP2 target engagement determines quantity of BWL. For these studies we used the dose that produced similar BWL for the three compounds
(7\%-11\% BWL after 7 days of repeat dosing). At these doses we observed similar target engagement profile and efficacy for all three compounds (Supplemental Fig. 6). These initial data suggest that MetAP2 target engagement can be used to predict efficacy for multiple chemical classes of MetAP2 inhibitors.

Translational Models and Prediction of Clinical Efficacy. Beloranib is a MetAP2 inhibitor that has been evaluated for body weight reduction in escalating-dose clinical trials with obese individuals (Shin et al., 2010; Hughes et al., 2013; Kim et al., 2015; https://zafgen.gcs-web.com/newsreleases/news-release-details/zafgen-refocuses-resourcesdevelopment-differentiated-second). To investigate if our obese mouse PK-PD-E models could predict human efficacy, we conducted further analyses using beloranib so that data comparisons could be made between mice and humans. We treated DIO mice at various doses of beloranib (Fig. 6A). Using a twice weekly dose schedule, approx. $10 \%$ weight loss was observed for both 0.1 and $0.3 \mathrm{mg} / \mathrm{kg}$ doses and a greater efficacy of approx. $17 \%$ was observed at $1 \mathrm{mg} / \mathrm{kg}$. However, if given daily, the $0.1 \mathrm{mg} / \mathrm{kg}$ dose produced approx. $25 \%$ weight loss. These body weight changes are not linear with respect to total weekly dose. To understand this better, we built dynamic PK and PK-E models relating BWL to plasma concentration of beloranib over time (Materials and Methods, Supplemental Fig. 3). From these models we simulated the average weekly beloranib exposure and BWL for twice-weekly and daily dosing regimens over a range of doses in DIO mice (Fig. 6B). The model predicted that efficacy saturates at each dose and that increasing the dose frequency yields greater reduction in body weight.

We next determined the relationship between inhibitor-bound MetAP2 and BWL in mice treated with beloranib using an occupancy assay (see Materials and Methods). We used the occupancy assay not only to evaluate a second method of MetAP2 target engagement but also to obtain additional quantitative data on how much MetAP2 needs to be inhibitor-bound to cause downstream effects. Using this assay we determined that MetAP2 is maximally occupied with inhibitor (approx. 100\%) from 2 to 8 hours post-dose in blood from DIO mice treated with $0.1 \mathrm{mg} / \mathrm{kg}$ beloranib (Fig. 7A). Occupancy decreased to approx. $85 \%$ at 24 hours and approached vehicle levels at 48 hours postdose. We measured the level of MetAP2 occupancy and concentration of beloranib at various doses (Fig. 7A; Supplemental Fig. 3; Supplemental Table 4) so that a dynamic PK-PD model could be generated (Materials and Methods, Supplemental Fig. 4). From this model we simulated the average weekly beloranib exposure and the average MetAP2 occupancy for twice-weekly and daily dosing regimens over a range of doses in DIO mice (Fig. 7B). MetAP2 occupancy saturated at submaximal levels for the less frequent schedule, providing deeper insight into the schedule effect on efficacy. Using these data, we also evaluated MetAP2 occupancy versus efficacy in obese mice, and as shown in Fig. 7C (diamonds), a correlation can be observed between occupancy and efficacy, with the more frequent dose schedule resulting in greater occupancy and efficacy.

Finally, we back-translated the beloranib efficacy data observed in the clinic to compare results in humans and mice (orange circles in Fig. 6B; Fig. 7, B and C). First, we calculated the mouse-equivalent exposures for the doses given to humans (0.6, 1.2, and $2.4 \mathrm{mg}$ ) using the reported human plasma concentration, see (Materials and Methods and Hughes et al., 2013). We then plotted the corresponding human weight loss achieved after 

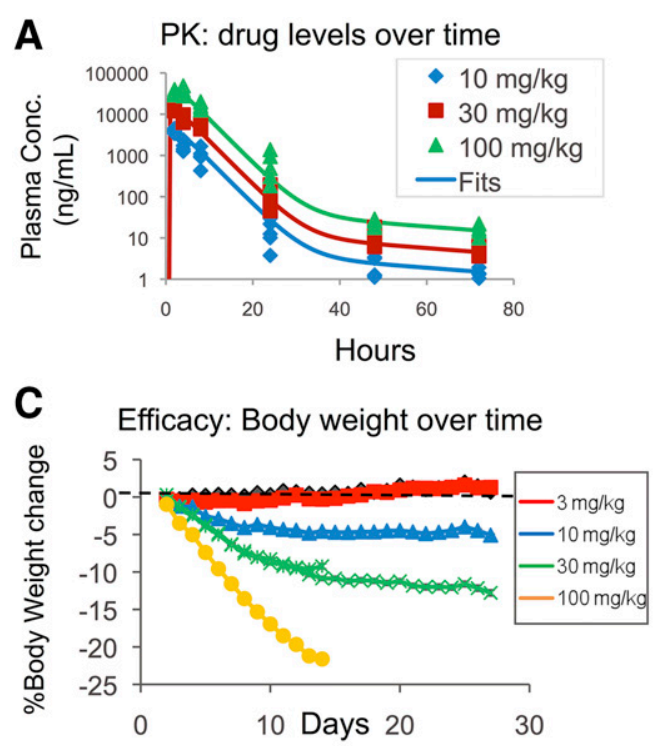

B PD: NMet 14-3-3y levels over time

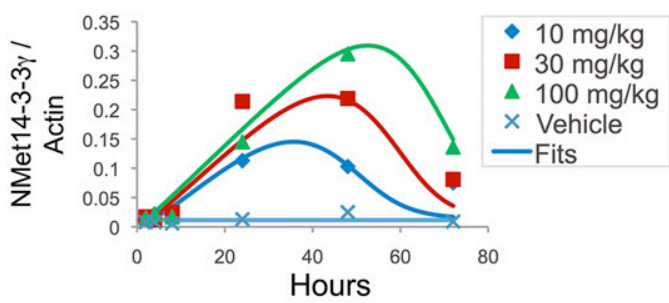

D Simulated PD: NMet 14-3-3y over time

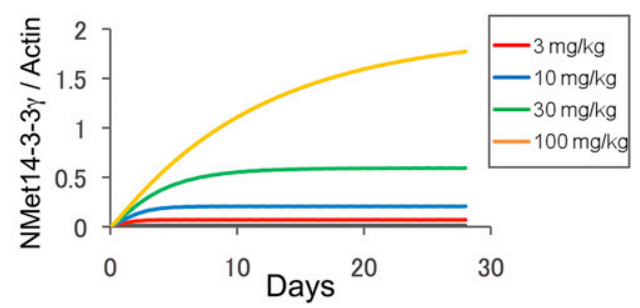

Fig. 4. Pharmacokinetics, target engagement pharmacodynamics, and efficacy (E) for compound 1 over time. (A) Total drug concentrations in plasma of DIO mice orally administered compound 1 at the indicated dose and time $(n=5$ mice per time point). Each colored symbol represents one mouse. (B) NMet14-3-3 $\gamma$ levels over time in PBMCs isolated from DIO mice administered a single dose of compound 1 at the indicated dose level. Each point represents NMet14-3-3 $\gamma$ levels in PBMCs isolated from blood that was pooled from five mice. (C) BWL studies over time were conducted in DIO mice orally administered vehicle or compound 1 at the indicated doses, daily for 14 or 28 days $(n=8$ or 12 mice per group, respectively). Each colored symbol represents one mouse. (D) NMet14-3-3 $\gamma$ levels in response to compound 1 were simulated over 28 days using the PK-PD model generated from $(\mathrm{A})$ and $(\mathrm{B})$.

12 weeks against the mouse-equivalent beloranib concentrations (Fig. 6B, orange circle symbols). Likewise, we simulated the average MetAP2 occupancy for the human regimens at the mouse-equivalent exposures (Fig. 7, B and C, orange circles). The PD-E model predicted that an average MetAP2 occupancy of approx. $27 \%$ produces approx. $10 \%$ BWL with a twice-weekly 2.4-mg dose. The relationship between MetAP2 occupancy and BWL in mice suggested that a lower dose level given daily would yield more MetAP2 occupancy and more efficacy than a higher dose given twice-weekly (see arrows Fig. 7C for low daily dose vs. high BIW dose that produce the same average beloranib concentration). Thus, MetAP2 target engagement may serve as a predictive biomarker of BWL efficacy as well as provide clinically actionable hypotheses (e.g., dose schedule for optimal efficacy) for the improvement of obesity treatment.

\section{Discussion}

In this study we demonstrate that 1) biomarkers, such as NMet14-3-3 $\gamma$ and inhibitor-bound MetAP2 can be used in a quantitative manner to determine level of MetAP2 engagement, 2) MetAP2 target-engagement biomarkers can be used to select potent compounds and predict BWL efficacy, and 3) daily administration of MetAP2 inhibitors at lower dose levels can be more efficacious than higher doses on a less frequent dose schedule (e.g., Fig. 6A). Our studies describe two biomarkers for target engagement, NMet14-3-3 $\gamma$ and inhibitor-bound MetAP2. The target engagement in freshly isolated PBMCs from donors is of particular interest, as it supports the use of NMet14-3-3 $\gamma$ as a biomarker in clinical studies for evaluation of obese patients treated with MetAP2 inhibitors. Thus, to extend our findings we investigated MetAP2 target engagement in PBMCs isolated from obese patients (Supplemental Table 3). The beloranib $\mathrm{EC}_{50}$ values were similar in PBMCs from lean and obese donors, suggesting the sensitivity of MetAP2 to beloranib in the peripheral compartment is not affected by obesity and confirming the potential for using NMet14-3-3 $\gamma$ to evaluate level of MetAP2 target engagement in obese patients treated with a MetAP2 inhibitor. A consideration for clinical application is the time to
A

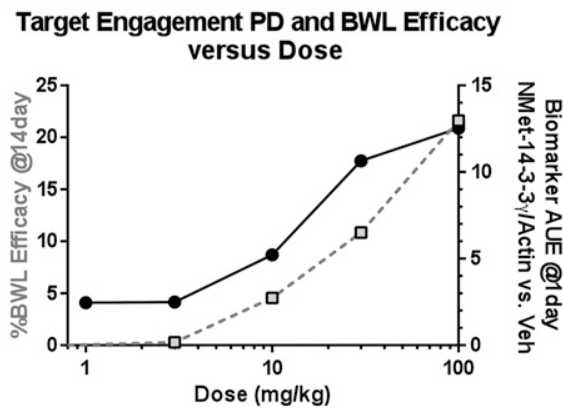

B

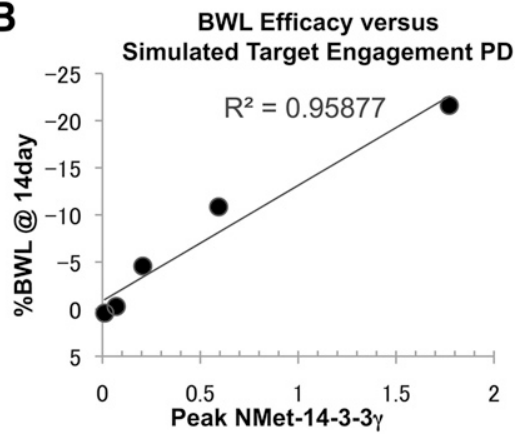

Fig. 5. Predicting efficacy of a MetAP2 inhibitor using target engagement PD. (A) Overlay of BWL efficacy (gray squares) determined after 14 days of oral daily dosing of compound 1 to DIO mice vs. MetAP2 target engagement PD (black circles) determined after one dose of compound 1 in mouse PBMCs. For PD, each circle represents blood pooled from six mice and for BWL each square symbol represents the average of $\geq 8$ mice. (B) PD-efficacy relationship for compound 1 using the BWL from Fig. 4C and simulated PD shown in Fig. 4D. 
A DIO Efficacy on two dose schedules

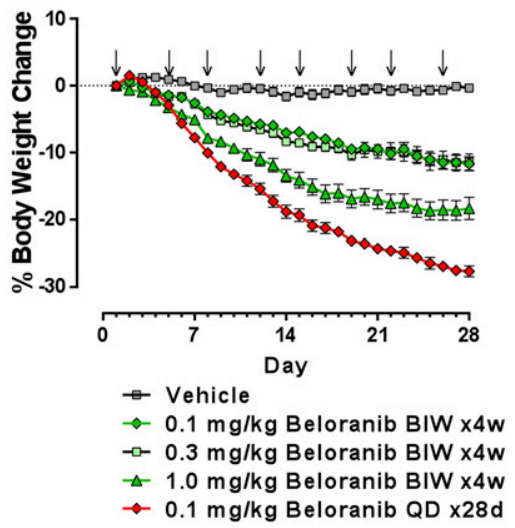

B

Translational PK/E Model

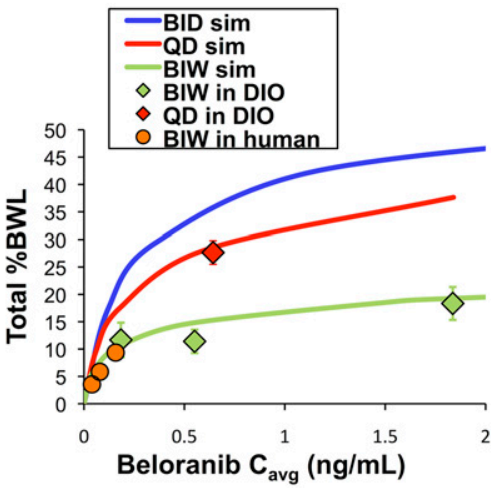

Fig. 6. A translational model for predicting efficacy of MetAP2 inhibitors in obese humans. (A) The BWL efficacy for beloranib was determined at several doses and two dose schedules in DIO mice $(n=8$ mice per group): twice weekly for 4 weeks [twice weekly (BIW) $\times$ $4 \mathrm{w}$ ] or daily for 28 days $(\mathrm{QD} \times 28 \mathrm{~d})$. Each symbol represents the average body weight change of eight mice at the dose and schedule indicated in the legend. (B) BWL at day 28 is plotted against average beloranib concentration simulated from the DIO PK model. The green and red symbols represent the corresponding dose groups from (A), whereas the orange symbols correspond to the BWL reported for human subjects at the mouse-equivalent exposure (Materials and Methods). PK-E curves were simulated (sim) for twice-weekly (BIW), daily (QD), or twice-daily (BID) administration. collect samples. While compound levels can be detected in plasma within a few minutes after a single administration of compound, NMet14-3-3 $\gamma$ levels were not detected until 8 hours post-dose (Fig. 4B). This slow induction may reflect the rate of new protein synthesis, because MetAP2 is localized to ribosomes where it cleaves off the initiator methionine on nascent
A Beloranib
PK and Receptor Occupancy

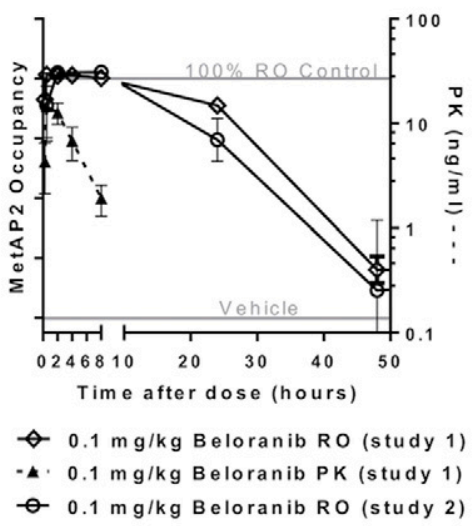

C

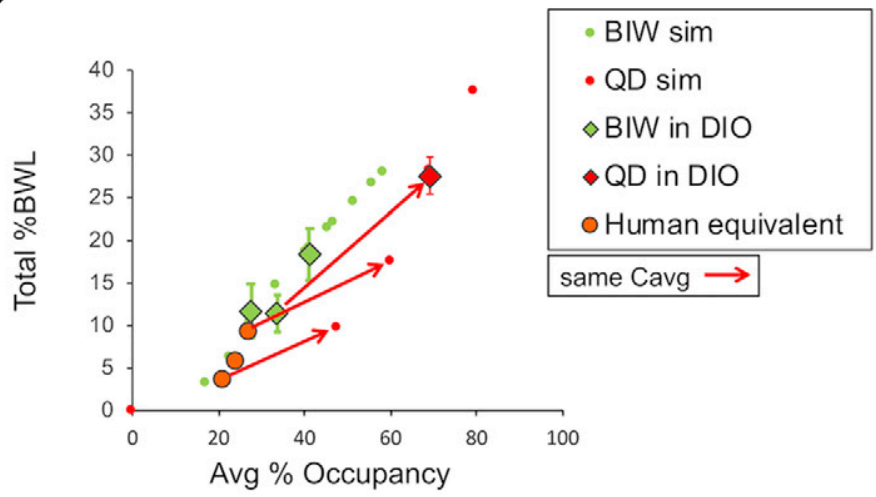

B

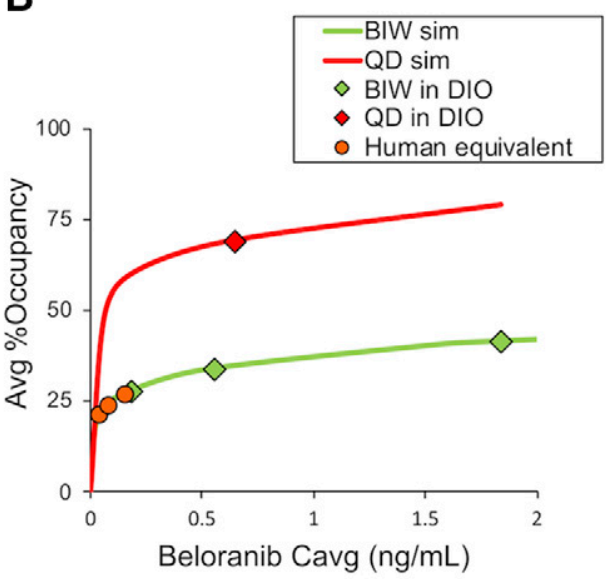

Fig. 7. Fraction of MetAP2 occupied by inhibitor in obese mice and humans. (A) The level of MetAP2 bound to beloranib over time in blood from DIO mice administered a single dose $(0.1 \mathrm{mg} / \mathrm{kg}$ given s.c. $)$ was determined using an ELISA. Each point represents the average \pm S.E.M. for five mice. Two independent in vivo studies are shown (solid lines) for receptor occupancy (RO). Drug levels over time in plasma are shown from one study (right $y$-axis and dotted line). The 100\% RO control was determined by adding saturating concentrations of Beloranib ex vivo to naive mouse blood, and the vehicle line represents mice dosed with vehicle. (B) Average simulated MetAP2 occupancy from the DIO PK-PD model plotted against the average simulated beloranib concentration. Lines indicate average MetAP2 occupancy simulated using the beloranib PK-PD model (Supplemental Fig. 4; Supplemental Table 1) for a range of doses on two different regimens in DIO mice [twice weekly (BIW) or daily (QD)]. The green and red symbols represent the simulations at the corresponding mouse dose groups from Fig. 6B, whereas the orange symbols correspond to the drug levels published from humans. (C) Backtranslation and simulated level of MetAP2 occupied in obese individuals who achieved weight loss. Using the data from Figs. $6 \mathrm{~B}$ and $7 \mathrm{~B}$, we developed the beloranib occupancy-efficacy model shown here relating the PK-E and PKPD model simulations on the same dosing regimens. Diamonds correspond to the dose groups in the mouse beloranib efficacy study, orange circles correspond to the human BWL observed clinically at the simulated MetAP2 occupancy, and the red and green circles are simulated points using the model as a basis. Red arrows demonstrate the predicted effect for switching to the more frequent daily schedule while maintaining the same average beloranib exposure $\left(\mathrm{C}_{\text {avg }}\right)$. More frequent lower doses are predicted to achieve more occupancy and BWL than less frequent higher doses. 
proteins. In addition, because of the slow decline of NMet14-3$3 \gamma$, it could be detected at 2 hours post-dose after multiple doses were given (Fig. 3). Overall, our investigations indicated that using collection time points that capture the rise and fall of biomarker and using the area under the PD effect curve produced the best correlation with efficacy (Fig. 5).

In our analyses we found that there is a strong schedule dependence on efficacy and that highest efficacy is associated with sustained low levels of unoccupied MetAP2. A lower level of efficacy is observed for less frequent dose schedules, e.g., twice weekly for beloranib (Fig. 6). The efficacy observed with less frequent schedules may be explained by the relatively long half-life of target engagement. The long half-life of the beloranib-bound MetAP2 is consistent with studies done in vitro to determine the half-life of fumagillin-bound MetAP2 (Datta et al., 2004). The complete turnover of both target engagement biomarkers (NMet14-3-3 $\gamma$ and inhibitor-bound MetAP2) seems to require greater than 24 hours after peak levels are observed as shown in Figs. 4B and 6A. This may explain why efficacy can be observed when drug is given only two times a week and why the more robust efficacy is achieved with daily dosing, which is associated with sustained target engagement. When the half-life of target engagement far outlasts the half-life of the drug (as is the case for both beloranib and compound 1), it is valuable to use target engagement biomarkers in addition to drug concentration to make decisions about dose and dose frequency for achieving efficacy. The data and models presented here suggest that the greater level of unoccupied MetAP2 between doses for the twice weekly dosing may explain the lower efficacy versus the daily dose that yields the same average beloranib exposure. Comparing the relationship between BWL and average MetAP2 occupancy (Fig. 7C) between the two dose schedules shows a clear advantage to the daily schedule and further verifies the value of MetAP2 engagement as a predictive efficacy biomarker.

Both target engagement biomarker assays described here are compatible with human PBMCs. This supports their potential use in clinical trials to: assess the level of active drug in blood, set efficacious doses, and obtain proof of mechanism. Each assay, however, has limitations that should be considered in their application. For example, although we and others demonstrate that the LI-COR Odyssey infrared imaging system for NMet14-3-3 $\gamma$ Western blot can be quantitative (Towbin et al., 2003; Schutz-Geschwender et al., 2004; Morgen et al., 2016), it does require reliable quality controls, precision, and attention to detail for consistency from study to study. Our cellular NMet14-3-3 $\gamma \quad \mathrm{EC}_{50}$ data for MetAP2 inhibitors indicated some assay-to-assay variability (Fig. 2), which prompted us to obtain multiple results for confident decision making. The MetAP2 occupancy ELISA assay is an alternate option for quantifying MetAP2 target engagement; though, this assay in its current format was only used for covalent MetAP2 inhibitors. Despite these limitations, we have used these biomarker assays successfully to demonstrate the concept that MetAP2 target engagement correlates quantitatively with BWL efficacy. Additionally, the rise and fall of NMet14-3-3 $\gamma$ in PBMCs from treated obese mice is similar to adipose (Supplemental Fig. 6), the tissue that probably contributes efficacy (Rupnick et al., 2002; Lijnen et al., 2010; Vath, 2010; Rohn, 2011; White et al., 2012; Huang et al., 2019). We also found that NMet14-3-3 $\gamma$ could be detected in PBMCs from obese patients after ex vivo treatment of blood and that the induction of NMet14-3-3 $\gamma$ in vitro and in DIO mice was observed with multiple chemically diverse MetAP2 inhibitors. Altogether, our findings provide rationale for using target engagement biomarkers in blood to predict efficacy in obese patients treated with MetAP2 inhibitors.

We have built two PD-E models that provide a starting point toward understanding the amount of target engagement needed to result in body weight reduction efficacy (Figs. 5 and 7). The PK, PD, and efficacy results showed that the plasma levels of compound can predict target engagement, which can predict efficacy in treated obese mice. The backtranslation of clinical beloranib data suggests that similar models may be applied to humans; however, to test how well these models predict human results, the evaluation of MetAP2 target engagement biomarkers versus disease outcome in dose escalating clinical trials is essential.

In summary, our analyses indicate that MetAP2 target engagement biomarkers can be used to select efficacious compounds and predict body weight loss in obese individuals treated with MetAP2 inhibitors. The preclinical studies showing the predictive nature of MetAP2 target engagement for weight loss efficacy and the comparison with backtranslated beloranib clinical data add to the biologic rationale for MetAP2 inhibition mechanism as a therapy for weight loss. Although our studies point toward future chemical design that focuses on maintaining MetAP2 occupancy (long residence time on MetAP2), other key factors to consider are high selectivity for MetAP2 in the adipose target tissue, safety, and convenient route of administration. Though our models show that a lower dose given more frequently has advantages for occupancy and efficacy versus a higher dose given less frequently, it is important to test if this regimen results in any potential advantage for the safety profile (owing to lower drug $\mathrm{C}_{\text {max }}$ upon each dose) or disadvantage (owing to more frequent dosing). Because each chemical entity is unique it will be important to demonstrate that long-term safety as well as efficacy in humans is achievable for the compounds that advance to clinical studies.

\section{Acknowledgments}

The authors thank Jocelyn Staunton, Min Lu, Brandi Bailey, Melinda Manuel, and Lisa Berquist for assistance in data generation; Haixia Wang, Mark Sabat, and Robert Gene for development of tool compounds and antibody reagents; and Munjal Patel, Teruk Hamada, and Mayankbhai Patel for assistance in generating and organizing the modeling files.

\section{Authorship Contributions}

Participated in research design: Farrell, Pachori, Zopf, Plonowski, Larson.

Conducted experiments: Balakrishna, Bilakovics, Fanjul, Holub, Matuszkiewicz, Rolzin, Banerjee, Ermolieff, Bartkowski, Mazur.

Contributed new reagents or analytic tools: Cheruvallath, McBride. Performed data analysis: Farrell, Zopf, Pachori, Plonowski.

Wrote or contributed to the writing of the manuscript: Farrell, Zopf, Huang.

\section{References}

Baranova A, Collantes R, Gowder SJ, Elariny H, Schlauch K, Younoszai A, King S, Randhawa M, Pusulury S, Alsheddi T, et al. (2005) Obesity-related differential gene expression in the visceral adipose tissue. Obes Surg 15:758-765.

Bråkenhielm E, Cao R, Gao B, Angelin B, Cannon B, Parini P, and Cao Y (2004) Angiogenesis inhibitor, TNP-470, prevents diet-induced and genetic obesity in mice. Circ Res 94:1579-1588. 
Cheruvallath Z, Tang M, McBride C, Komandla M, Miura J, Ton-Nu T, Erikson P, Feng J, Farrell P, Lawson JD, et al. (2016) Discovery of potent, reversible MetAP2 inhibitors via fragment based drug discovery and structure based drug design-Part 1. Bioorg Med Chem Lett 26:2774-2778.

Datta B, Majumdar A, Datta R, and Balusu R (2004) Treatment of cells with the angiogenic inhibitor fumagillin results in increased stability of eukaryotic initiation factor 2-associated glycoprotein, p67, and reduced phosphorylation of extracellular signal-regulated kinases. Biochemistry 43:14821-14831.

Foster GD, Wadden TA, Vogt RA, and Brewer G (1997) What is a reasonable weight loss? Patients' expectations and evaluations of obesity treatment outcomes. $J$ Consult Clin Psychol 65:79-85.

Hageman RS, Wagener A, Hantschel C, Svenson KL, Churchill GA, and Brockmann GA (2010) High-fat diet leads to tissue-specific changes reflecting risk factors for diseases in DBA/2J mice. Physiol Genomics 42:55-66.

Hong CI, Kim JW, Lee SJ, Ahn SK, Choi NS, Hong RK, Chun HS, Moon SK, and Han CK (2000) inventors, Chong Kun Dang Corporation (KR), assignee. Fumagillol derivatives and processes for preparing the same. U.S. patent 6,063,812. 2000 May 16.

Huang HJ, Holub C, Rolzin P, Bilakovics J, Fanjul A, Satomi Y, Plonowski A, Larson CJ, and Farrell PJ (2019) MetAP2 inhibition increases energy expenditure through direct action on brown adipocytes. $J$ Biol Chem 294 9567-9575

Hughes TE, Kim DD, Marjason J, Proietto J, Whitehead JP, and Vath JE (2013) Ascending dose-controlled trial of beloranib, a novel obesity treatment for safety, tolerability, and weight loss in obese women. Obesity (Silver Spring) 21:1782-1788,

Kim DD, Krishnarajah J, Lillioja S, de Looze F, Marjason J, Proietto J, Shakib S, Stuckey BGA, Vath JE, and Hughes TE (2015) Efficacy and safety of beloranib for weight loss in obese adults: a randomized controlled trial. Diabetes Obes Metab 17: $566-572$.

Kim YM, An JJ, Jin YJ, Rhee Y, Cha BS, Lee HC, and Lim SK (2007) Assessment of the anti-obesity effects of the TNP-470 analog, CKD-732. J Mol Endocrinol 38: 455-465.

Klimcáková E, Roussel B, Márquez-Quiñones A, Kovácová Z, Kováciková M, Combes M, Siklová-Vítková M, Hejnová J, Srámková P, Bouloumié A, et al. (2011) Worsening of obesity and metabolic status yields similar molecular adaptations in human subcutaneous and visceral adipose tissue: decreased metabolism and increased immune response. J Clin Endocrinol Metab 96:E73-E82.

Lijnen HR, Frederix L, and Van Hoef B (2010) Fumagillin reduces adipose tissue formation in murine models of nutritionally induced obesity. Obesity (Silver Spring) 18:2241-2246.

Liu S, Widom J, Kemp CW, Crews CM, and Clardy J (1998) Structure of human methionine aminopeptidase-2 complexed with fumagillin. Science $\mathbf{2 8 2}$ 1324-1327.

McBride C, Cheruvallath Z, Komandla M, Tang M, Farrell P, Lawson JD, Vanderpool D, Wu Y, Dougan DR, Plonowski A, et al. (2016) Discovery of potent, reversible MetAP2 inhibitors via fragment based drug discovery and structure based drug design-Part 2. Bioorg Med Chem Lett 26:2779-2783.
Morgen M, Jöst C, Malz M, Janowski R, Niessing D, Klein CD, Gunkel N, and Miller AK (2016) Spiroepoxytriazoles are fumagillin-like irreversible inhibitors of MetAP2 with potent cellular activity. ACS Chem Biol 11:1001-1011.

Morrison JF (1982) The slow-binding and slow, tight-binding inhibition of enzymecatalysed reactions. Trends Biochem Sci 7:102-105.

Ogden C, Carroll M, Fryar C, and Flegal K (2015) Prevalence of obesity among adults and youth: United States, 2011-2014. NCHS Data Brief No. 219:1-8.

Rohn J (2011) Newsmaker: Zafgen. Nat Biotechnol 29:1068.

Rosell M, Kaforou M, Frontini A, Okolo A, Chan YW, Nikolopoulou E, Millership S, Fenech ME, MacIntyre D, Turner JO, et al. (2014) Brown and white adipose tissues: intrinsic differences in gene expression and response to cold exposure in mice. Am J Physiol Endocrinol Metab 306:E945-E964.

Rupnick MA, Panigrahy D, Zhang CY, Dallabrida SM, Lowell BB, Langer R, and Folkman MJ (2002) Adipose tissue mass can be regulated through the vasculature. Proc Natl Acad Sci USA 99:10730-10735.

Schutz-Geschwender A, Zhang Y, Holt T, McDermitt D, and Olive DM (2004) Quantitative, two-color western blot detection with infrared fluorescence. LI-COR Biosciences. 1-7.

Shin SJ, Jeung HC, Ahn JB, Rha SY, Roh JK, Park KS, Kim DH, Kim C, and Chung HC (2010) A phase I pharmacokinetic and pharmacodynamic study of CKD-732, an antiangiogenic agent, in patients with refractory solid cancer. Invest New Drugs 28: $650-658$.

Towbin H, Bair KW, DeCaprio JA, Eck MJ, Kim S, Kinder FR, Morollo A, Mueller DR, Schindler P, Song HK, et al. (2003) Proteomics-based target identification bengamides as a new class of methionine aminopeptidase inhibitors. J Biol Chem 278:52964-52971.

Vath J (2010) Methods of treating an overweight or obese subject, US 2010/0016425 A1. Jan 21, 2010, Inventor James Vath, Lynnfield, MA, Assignee Zafgen, Inc Cambridge, MA.

Wang J, Sheppard GS, Lou P, Kawai M, BaMaung N, Erickson SA, Tucker-Garcia L, Park C, Bouska J, Wang YC, et al. (2003) Tumor suppression by a rationally designed reversible inhibitor of methionine aminopeptidase-2. Cancer Res 63 7861-7869.

White HM, Acton AJ, and Considine RV (2012) The angiogenic inhibitor TNP-470 decreases caloric intake and weight gain in high-fat fed mice. Obesity (Silver Spring) 20:2003-2009.

Yanovski SZ and Yanovski JA (2014) Long-term drug treatment for obesity: a systematic and clinical review. JAMA 311:74-86.

Zhou GC, Liu F, Wan J, Wang J, Wang D, Wei P, and Ouyang P (2013) Design, synthesis and evaluation of a cellular stable and detectable biotinylated fumagillin probe and investigation of cell permeability of fumagillin and its analogs to endothelial and cancer cells. Eur J Med Chem 70:631-639.

Address correspondence to: Dr. Pamela J. Farrell, Takeda California, 9625 Towne Center Drive, San Diego, CA 92121. E-mail: m.farrell@att.net 